\title{
A PULSE CALIBRATION SYSTEM FOR THE VLBA
}

\section{A. R. THOMPSON}

National Radio Astronomy Observatory, Edgemont Road, Charlottesville, VA.

D. S. BAGRI

National Radio Astronomy Observatory, Socorro, NM.

\begin{abstract}
In pulse calibration of an interferometer a series of short pulses is inserted into the receiver input to produce a spectrum of equi-spaced lines. The use of these for calibration, and the proposed system for the VLBA, are described.
\end{abstract}

Geodetic and astrometric measurements using VLBI depend upon absolute measurements of the phase- or delay-differences of the signals from a radio source at the different receiving antennas. By the use of water-vapor radiometers, dual frequency observing, or making differential measurements between closely spaced sources, the effects of the atmosphere can be reduced to a level at which calibration of instrumental phase and delay is desirable to an accuracy of order 3 ps (corresponding to one $\mathrm{mm}$ of space path). Variations in the receiving system result from temperature variations, cable flexing, etc. at each antenna. Calibration of these effects by injecting a signal at the input of a receiver and measuring the phase of the resulting baseband component has been successfully practiced in VLBI since the mid-1970's. In the system developed by Rogers (Whitney et al. 1976) and widely used in the Mark III VLBI system (Clark et al. 1985), the injected signal is a train of pulses of width approximately 25 ps at intervals of $1 \mu \mathrm{s}$. The local oscillator frequencies are set so that in each baseband channel one of the injected harmonics of $1 \mathrm{MHz}$ appears at a frequency of $10 \mathrm{kHz}$. The 10 $\mathrm{kHz}$ components are extracted at the correlator where their phases are measured by multiplying each baseband output with two $10 \mathrm{kHz}$ signals in phase quadrature. The hydrogen maser at the antenna provides the timing standard, and a reference frequency of $5 \mathrm{MHz}$ is transmitted by cable to the pulse generator near the antenna feed. The electrical length of the cable is constantly monitored by a round-trip phase measurement based on the method of Swarup and Yang (1961) using a modulated reflector. A gating switch at the pulse generator output passes only one positive pulse per $\mu \mathrm{s}$ from 
the tunnel diode. The pulse generator and the cable through which the pulses are injected into the front end must be stable in delay to less than a few ps.

In a VLBI system such as the Mark III or the VLBA (Kellermann and Thompson 1985), a number of baseband channels are distributed across each observing band, so changes in the instrumental phase path can be monitored at a number of frequencies, and corrections applied in the data reduction. Changes that are constant across the frequency band indicate variations in the phase of a local oscillator, changes that vary linearly with frequency indicate variation in time delay in the instrumental path, and changes that are higher order functions of frequency indicate more complex effects such as temperature related variations in filter responses.

In the VLBA the electronic system is specifically designed for good phase stability, but a pulse calibration system should improve the performance still further. There are two reasons for this expectation. First, the pulse generator is physically small and simple compared with the receiving system which contains numerous components distributed between the front end assembly, modules in the vertex room, and more modules in the electronics building, at each antenna. Second, the analog circuit components in the pulse generator have broadband frequency responses and relatively short time delays. In the VLBA receiving system the expected dependence of propagation time on temperature, based on experience with the VLA, varies from a few ps per ${ }^{\circ} \mathrm{C}$ for the front ends to a few hundred ps per ${ }^{\circ} \mathrm{C}$ for the baseband amplifiers (Thompson and Bagri 1988). The time-delay stability gets worse as the signals progress from the front ends to the baseband amplifiers, largely because the system bandwidth decreases. The phase stability, however, remains roughly constant because the frequency of the signal decreases from the observing frequency to baseband. In a typical wellregulated environment in which the temperature varies over a range of $\pm 1^{\circ} \mathrm{C}$, the overall instrumental delay may vary by several hundred ps. Delay variations resulting from flexing of cables in the antenna cable wrap vary with antenna pointing and thus would be difficult to calibrate astronomically.

A block diagram of the proposed VLBA pulse generator is shown in Fig. 1. The system contains four features that we believe will enhance the accuracy and flexibility. (1) The reference frequency transmitted from the maser is $500 \mathrm{MHz}$, rather than $5 \mathrm{MHz}$, which will improve the precision with which the length of the cable can be monitored. An accuracy of $1^{\circ}$ in the round-trip phase will then correspond to $2.7 \mathrm{ps}$ in oneway transmission delay in the cable. (2) The phase of the pulse generator output, rather than the input, is controlled by the reference signal from the maser, and the effect of any variation in the time delay in the pulse generator is 


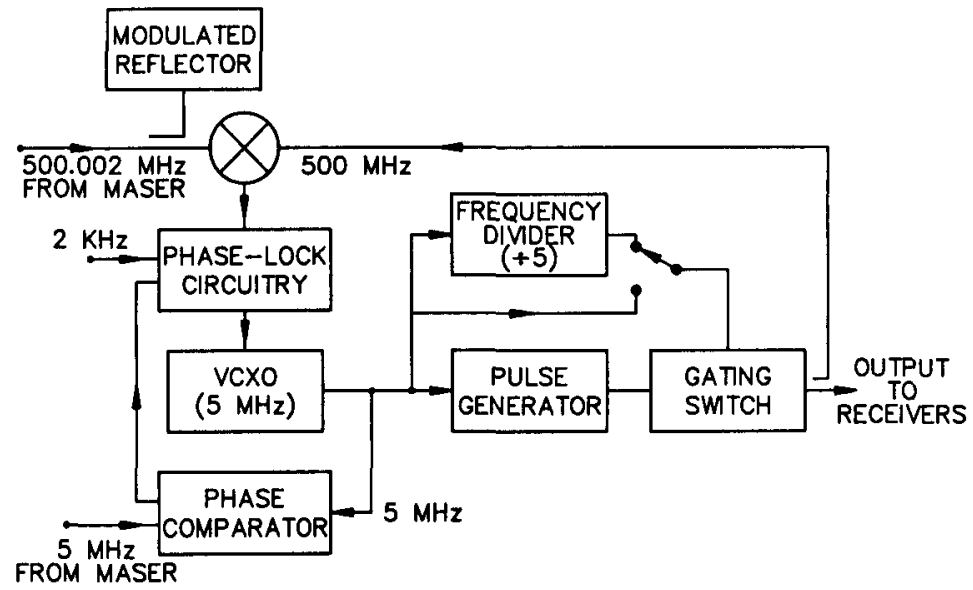

Fig. 1. Pulse Generator for the VLBA Antennas.

compensated by a phase-locked loop. This is accomplished by driving the pulse generator from a crystal controlled oscillator which is phase locked by comparing the phase of the output of the pulse generator at $500 \mathrm{MHz}$ with the $500 \mathrm{MHz}$ reference from the maser. To facilitate this phase comparison the reference from the maser is offset from $500 \mathrm{MHz}$ by approximately $2 \mathrm{kHz}$. In a similar scheme used in the VLA, in which the harmonic of a crystal oscillator is locked to a reference in the local oscillator system, the resulting phase stability was found to be as good as 1 ps per ${ }^{\circ} \mathrm{C}$. Because the oscillator in Fig. 1 is locked on its $100^{\text {th }}$ harmonic, lock points occur at intervals of $3.6^{\circ}$ in the phase of the $5 \mathrm{MHz}$ oscillator. To resolve the resulting phase ambiguity, the oscillator output is compared in phase with a $5 \mathrm{MHz}$ signal from the maser, and the loop is adjusted accordingly. (3) The gating switch can be driven to pass pulses at either 200 ns or $1 \mu$ s intervals, thereby producing lines at intervals of $5 \mathrm{MHz}$ or $1 \mathrm{MHz}$ respectively. In the spectrum with lines at $5 \mathrm{MHz}$ intervals the power level of each line is higher by a factor of 25 than that of a line in the spectrum with $1 \mathrm{MHz}$

intervals. Thus the $5 \mathrm{MHz}$-interval spectrum is useful for the higher frequency bands. (4) The frequencies at which the calibration signals can be extracted will be tunable across the full bandwidth of the baseband filters, which is variable from $62.5 \mathrm{kHz}$ to $16 \mathrm{MHz}$ in the VLBA. The tuning steps need be no narrower than the $10 \mathrm{kHz}$ tuning steps of the final local oscillator. With the wider bandwidths several calibration lines fall within a single baseband. The plan is to provide for measurement of one calibration signal from each baseband simultaneously, i.e. 16 extractors are needed for each antenna.

Certain baseband frequencies are to be avoided for 
monitoring of phase responses. For example, in the VLBA 10 $\mathrm{kHz}$ is used as the baseband local oscillator reference, and very low level spurious signals at this frequency could therefore infiltrate the electronics and cause errors in the phase calibration. Also, the local oscillators should be set so that the calibration frequencies do not occur at the same baseband frequency in the upper and lower sidebands for the same baseband converter, because of the finite image rejection in the baseband conversion. Thus if one frequency is extracted simultaneously from each baseband, at least two independent reference frequency generators are required. To provide somewhat more flexibility, four independent reference generators are planned. The stability of their outputs can be checked directly with respect to reference signals from the maser. The assignment of the extractors to the baseband signals will be variable to allow up to four signals to be extracted simultaneously from selected bands. Thus it will be possible to monitor variations in the phase response across the basebands.

The calibration extraction and measurement can be performed at any point after the samplers, at which the time of each data sample is preserved within the record. In the VLBA the phase measurement will be performed in real time at the antenna sites and recorded on the monitor tape. The calibration signals can also be measured at the correlator, but this will require a separate pass of the tapes through the playback recorders from that used to correlate the cosmic signals. When all effects are considered, we believe that it will be possible to monitor variations in the signal propagation times of the receiving system to within a few ps.

\section{REFERENCES,}

Clark, T. A., et al. 1985, Precision Geodesy Using the MarkIII Very-Long-Baseline Interferometer System, IEEE Trans. Geosci. Remote Sensing, GE-23, 438-449.

Kellermann, K. I. and Thompson, A. R. 1985, The Very Long Baseline Array, Science, 229, 123-130.

Swarup, G. and Yang, K. S. 1961, Phase Adjustment of Large Antennas, IEEE Trans. Antennas Propag., AP-9, 75-81.

Thompson, A. R. and Bagri, D. S. 1988, A Pulse Calibration Scheme for the VLBA, VLB Array Memo. No. 622.

Whitney, A. R., et al. 1976, A Very-Long-Baseline Interferometer System for Geodetic Applications, Radio Science, 11, 421-432. 
J. P. Hamaker: I have always wondered how these pulse calibration systems can work as well as they apparently do. Indeed, the phases of all those harmonics in the pulse train relative to each other and to the fundamental depend very critically on the detailed shape of the pulse and on the precise moment at which the pulse occurs, both of which must depend on temperatures, signal voltages, etc. Why is it that these dependencies are so much weaker than one might have feared?

Dick Thompson and D. Bagri: With the system in Fig.3, the phase of the $500 \mathrm{MHz}$ line is stabilized relative to the hydrogen maser frequency standard, and the effect of any time delay in the pulse generator is thereby removed. The relative phases of other lines should depend only on the pulse shape. With careful design, the driving waveform and the physical temperature of the pulse generator, and hence the pulse shape, should not vary greatly. In any case, the effective time variation for any frequency that results from pulse shape variation can be no more than a fraction of the $25 \mathrm{ps}$. pulse length. For example, 5 ps. at $8.4 \mathrm{GHz}$ corresponds to 15 degrees of phase. Future developments should lead to narrower pulses and thus improve the phase stability over the whole frequency range as well as extending the calibration to higher frequency bands. 